\section{Elección de escuela en sectores populares: Estado, mercado e integración social ${ }^{\star}$}

School choice in low income populations: State, market and social inclusion.

\author{
Cristián Bellei ${ }^{* *}$ \\ Manuel Canales ${ }^{* \star}$ \\ Víctor OrELLANA ${ }^{* \star \star *}$ \\ Mariana ConTRERAS ${ }^{\star \star \star * *}$
}

\begin{abstract}
Se agradece el financiamiento otorgado por Fondecyt 1130430 y Proyecto Basal FB0003, del Programa de Investigación Asociativa, ambos de Conicyt, Ministerio de Educación de Chile.
\end{abstract}

** Centro de Investigación Avanzada en Educación y Departamento de Sociología, Universidad de Chile; Periodista José Carrasco Tapia \#75, Santiago, Chile. Correo electrónico: cbellei@ ciae.uchile.cl

*** Departamento de Sociología, Universidad de Chile, Periodista José Carrasco Tapia \#75, Santiago, Chile. Correo electrónico: mcanalsc@gmail.com

**** Centro de Investigación Avanzada en Educación y Departamento de Sociología, Universidad de Chile; Periodista José Carrasco Tapia \#75, Santiago, Chile. Correo electrónico: victor.orellana@ ciae.uchile.cl

***** Centro de Investigación Avanzada en Educación y Departamento de Sociología, Universidad de Chile; Periodista José Carrasco Tapia \#75, Santiago, Chile. Correo electrónico: mdemarianacm@ gmail.com

\section{Resumen}

La libre elección de escuelas es considerada una pieza clave de las reformas de mercado en educación, especialmente cuando incluye la opción de escuelas privadas. Existe un amplio debate acerca de las consecuencias de estas dinámicas en las clases bajas, incluyendo si y cómo estos sectores participarán en la lógica de oferta-demanda que los proponentes del mercado educacional anticipan. Este artículo -basado en un estudio cualitativo en el cual se realizaron entrevistas y grupos de discusión en una zona popular prototípica de Santiago- busca comprender la elección escolar en la clase baja chilena. Los hallazgos permiten identificar dos tipos de electores: uno activo e intranquilo que lucha en la parte inferior del mercado educacional, y otro tradicional pragmático que permanece fuera del mercado, principalmente cobijado en la educación pública. Además, encontramos transversalmente una fuerte imagen social de la elección -para algunos la única real- de educación técnica-secundaria. Cada una de estas "elecciones" tiene para los padres de sectores populares una densa trama de significados, muy lejos de la caracterización pasiva del "no elector" de algunos estudios previos.

Palabras clave: elección de escuela, estudios cualitativos, sociología de la educación, mercado educacional

\begin{abstract}
Free school choice is regarded as a key component of market oriented educational reforms, especially when it incorporates private
\end{abstract}


schools. There is an important debate about the consequences of these dynamics for lower social classes, including whether and how low income families will participate in the supply-demand dynamics that market proponents envision. This paper -based on a qualitative study that included interviews and focus groups in a typical low income area of Santiago- attempts to understand the school choice process in the Chilean low social class. Our findings allowed us to identify two types of approaches to school choice: one active and anxious who is fighting in the lower section of the educational market; and another one traditional and pragmatic who remains outside the market, mainly covered by public education. Additionally, we found a generalized strong social view of vocational secondary education, for some parents the sole true "school choice" they have. Each of these "choices" have a thick and complex meaning for low income parents, which is very different from the view of "passive actors" coming from some previous research.

Key words: school choice, qualitative studies, sociology of education, educational market

\section{Introducción: clases bajas y mercado escolar}

Si los padres deben enviar a sus hijos a la escuela pública del barrio o si los estudiantes son asignados aleatoriamente a las escuelas disponibles, la pregunta por la "elección de escuela" por parte de las familias es casi un sinsentido. Sin embargo, en las últimas décadas ha adquirido creciente relevancia la idea de permitir y estimular que las familias elijan activamente la escuela para sus hijos. Por cierto, una fracción (variable entre países) de padres de ingresos altos históricamente ha elegido entre escuelas privadas de alto costo, manteniéndose al margen de la educación financiada con recursos públicos. Así, la expansión de la elección de escuela es presentada por sus proponentes como la democratización de un privilegio (Chubb y Moe 1990). Desde esta visión, el que las familias puedan decidir en qué escuela educar a sus hijos produciría un doble efecto virtuoso: los estudiantes asistirían a escuelas más apropiadas a sus características y más coherentes con la enseñanza familiar, y las escuelas ajustarían sus propuestas educativas para responder a las demandas de las familias y las particularidades de los alumnos. Aunque no es intrínseco a esta propuesta, su versión más difundida en la arena política busca en último término configurar un mercado privado de escuelas financiado por el estado (Gentili et al. 2009). Como se sabe, Chile es el país que ha ido más lejos en esta dirección.

Buena parte de la investigación social sobre elección de escuelas puede ser interpretada como un testeo de la teoría de la "acción racional" presupuesta por los proponentes del mercado educacional. El hallazgo principal de esta literatura es la centralidad que tienen para los padres los aspectos sociales en la elección de escuela, por encima de consideraciones propiamente académicas (Ball, Bowe y Gewirtz 1995; Butler y van Zanten 2007). El carácter social de esta elección presionaría por un aumento de la segregación escolar, por cuanto las clases altas y medias utilizarían sus mayores recursos económicos, sociales y culturales para distanciarse de las clases bajas (incluyendo en algunos contextos minorías étnicas e inmigrantes), asegurando para sus hijos circuitos aventajados de socialización, educación e inserción laboral. Sin embargo, la investigación social ha puesto menos énfasis en comprender 
estas dinámicas al interior de la clase baja. Los sectores populares tienden a ser vistos como los grandes perjudicados por la expansión de las dinámicas de libre elección (Gewirtz, Ball y Bowe 1996; Reay, Ball y Taylor 1997; Olmedo 2007; Felouzis, Maroy y Van Zanten 2013), al quedar "abandonados" en escuelas públicas, gratuitas y no selectivas. Al contrastarlos con las dinámicas observadas en las clases superiores, los sectores bajos han sido caracterizados como "no electores", connotando una actitud pasiva frente a las oportunidades que se les ofrecen.

Este artículo busca profundizar en la comprensión del fenómeno de la elección escolar en las clases bajas, indagando sobre las razones y consideraciones que ponen en juego las familias durante el proceso de decidir dónde educar a sus hijos. El caso chileno ofrece un escenario privilegiado para este estudio, por cuanto -desde 1980- su sistema de financiamiento basado en un voucher universal garantiza, en principio, una completa libertad de elección para los padres y fuerza a las escuelas públicas y privadas a competir por sus preferencias (Bellei 2015).

\section{La investigación sobre elección de escuela en sectores populares}

La investigación ha encontrado que la clase social es el principal atributo para comprender las elecciones escolares de los padres, pues estas dependen estrechamente de la posesión de recursos económicos, culturales y sociales (Ball 1993; Ball, Bowe y Gewirtz 1996; Broccolichi y Van Zanten 2000; Ball 2003; Devine 2004). Así, en dinámicas de "mercado escolar", los estudios convergen en señalar que las familias de clase baja se hallan en situación de desventaja. La posibilidad de elegir escuela no les garantiza mayor acceso a mejores oportunidades educativas, ya que se enfrentan al proceso electivo (incluyendo las prácticas de admisión escolar) con herramientas de información, conocimiento y redes sociales limitadas y escasamente valoradas, acentuándose su desventaja frente a las clases medias y alta (Gewirtz, Ball y Bowe 1996; Reay, Ball y Taylor 1997; Olmedo 2007; Felouzis, Maroy y Van Zanten 2013).

En efecto, aun cuando posean algún margen de decisión (en principio), la literatura muestra que los padres de menor escolaridad y menores ingresos orientan sus decisiones por criterios prácticos, privilegiando la cercanía de la escuela con respecto a sus hogares, hallazgo que ha sido reportado en muy diversos contextos nacionales (Waslander y Thrupp 1995; Roland 2012; Fernández 2004; Bernal 2005; Ball, Bowe y Gewirtz 1996; Ball \& Vincent 1998; Reay et al. 1997; Bosetti 2004; Ballion 1991; Whitsel 2014; Costa y Koslinski 2012). Estos padres valoran la formación de sus hijos en un clima escolar sano y el resguardo de su bienestar físico y emocional (Ball y Reay 1998; Felouzis, Maroy y Van Zanten 2013), privilegiando la familiaridad y cercanía de sus hijos con las amistades del barrio (Ball y Vincent 1998). De hecho, los padres de sectores populares preferirían para sus hijos escuelas sin gran exigencia académica, que les permitan luego continuar estudios secundarios vocacionales, con miras a una rápida y mejor inserción laboral (Bosetti 2004; Ballion 1991; Palheta 2012), autoexcluyéndose de escuelas más selectivas por temor al fracaso escolar (Rambla 2003; Ben Ayed 2011; Cartier et al. 2008).

Respecto al proceso de elección escolar, los padres de clase baja perciben una relación 
distante y jerárquica con las escuelas, ya que consideran no tener las competencias requeridas para buscar, evaluar y negociar con la oferta institucional (Alegre y Benito 2012b; Alegre, Benito y González 2014). Así, por ejemplo, la literatura es convergente en señalar que estos padres utilizan principalmente fuentes de información de tipo social, la cual les proporciona una manera que consideran fiable y comprensible de decodificar las escuelas (Ball y Vincent 1998). La fuerte dependencia de fuentes sociales de información y la relevancia que dan a la disciplina en el colegio hace que los padres de clases populares sean muy sensibles a los rumores que circulan sobre las escuelas (Felouzis y Perroton 2007; Felouzis, Maroy y Van Zanten 2013).

La aparente distancia entre el comportamiento de los padres de sectores populares y el prototipo de "elector de mercado", ha llevado a algunos investigadores a caracterizarles como pasivos y poco involucrados en el proceso de elección escolar. Así, por encontrarse fuera de la lógica de mercado, ha sido recurrente asignarles un perfil de "no electores" (Bernal 2005), "electores inertes" (Echols y Willms 1992) o "electores desconectados" (Gewirtz, Ball y Bowe 1995), dominados por la apatía y la resignación, y con escasa disposición a la búsqueda, comparación y elección activa. Una explicación algo distinta propone que los padres de clase baja serían "electores confiados", pues consideran que no existen grandes diferencias entre las escuelas, lo que los llevaría a permanecer en las escuelas públicas y cercanas a sus hogares (Alegre y Benito 2012a).

Una hipótesis alternativa, sin embargo, arranca de la constatación fundamental antes referida: que la elección escolar está estrechamente vinculada a la clase social: a las familias de sectores populares les resultaría imposible replicar la forma "activa" de participación en el mercado escolar, identificada con la conducta de las clases altas y medias. Los padres de clase baja serían, más bien, electores pragmáticos, quienes conscientes de sus limitaciones y desventajas frente a otras familias en el campo educacional, adecuarían sus expectativas a sus posibilidades reales de éxito (cálculo a su vez marcado por sus propias experiencias educacionales no-exitosas). Sus decisiones estarían signadas por la incertidumbre, el miedo al fracaso escolar y el reconocimiento de limitantes que les impedirían proyectarse más allá de sus fronteras de clase, configurándose una "evasión racional de decisiones de alto riesgo" (Reay, Ball y Taylor 1997). Esta interpretación es relevante porque apunta a reconocer que el "mercado educacional" supone ciertas bases socioculturales para su operación, al tiempo que recuerda que la estratificación basada en clases sociales no se reduce a la mera diferencia de recursos económicos entre las familias. Esta es la perspectiva que asumimos en este artículo para interpretar el caso chileno.

Si bien la investigación cualitativa sobre elección de escuela en Chile es relativamente escasa, sus hallazgos básicos sobre los sectores populares son bastante convergentes con la literatura internacional, aunque con algunas particularidades. La cercanía al hogar fue identificada tempranamente como una variable relevante en la elección escolar de los sectores populares (Espínola 1983); aunque, al aumentar el nivel de escolaridad de los padres, adquirirían relevancia otros elementos como la satisfacción, la infraestructura o la "calidad" (Navarro 2004; Córdoba 2014). La familiaridad con el establecimiento (dada por la experiencia 
directa o de familiares) es también un criterio importante de decisión (Navarro 2004; Raczynski y Hernández 2011). Adicionalmente, los padres de clase baja ponen mucha atención en la seguridad y protección de sus hijos al interior de la escuela (Navarro 2004; Córdoba 2014), evitando establecimientos en que perciben una alta concentración de estudiantes con problemas conductuales o provenientes de familias consideradas marginales (Espínola 1983; Raczynski y Hernández 2011; Córdoba 2014; Carrasco, Falabella y Mendoza 2015). En efecto, en lo esencial, la noción de "calidad" educativa de los padres de clase baja estaría vinculada con aspectos como el orden, la disciplina y el ambiente de respeto en las escuelas, postergando a un segundo plano el aprendizaje y logro académico.

Finalmente, si bien los padres chilenos de sectores populares también se informan de las escuelas principalmente por sus redes sociales, ellos utilizarían dos "indicadores" adicionales (Navarro 2004; Raczynski y Hernández 2011; Córdoba 2014): primero, la observación directa, para indagar sobre la conducta y apariencia de los estudiantes, o comparar los cuadernos de alumnos de diferentes escuelas; y segundo, el precio de las escuelas, que, al actuar como dispositivo de segmentación social de la demanda, se le atribuye un efecto selectivo sobre el estudiantado. Interesantemente, esta observación no se reduciría al momento de la elección y muchos padres mantendrían una cierta "vigilancia", manifestando una alta disposición al cambio de escuela ante la insatisfacción (Raczynski y Hernández 2011; Córdoba 2014). Así, Córdoba (2014) enfatiza que los padres de sectores pobres sí intentan escoger "la mejor escuela" a su alcance, aun cuando sus únicas opciones sean públicas o privadas gratuitas.
En suma, los estudios sobre familias de clase baja concluyen que en la dinámica de elección escolar priman consideraciones sociales y prácticas en la elección, por encima de la preocupación por la "calidad educativa", entendida en sentido estricto. Los hallazgos indican que, si bien los sectores populares chilenos no se comportan bajo el precepto teórico del mercado escolar, tampoco tienen una conducta pasiva. Comprender tal fenómeno en un sentido positivo (superando las nociones de "no elector") requiere profundizar esta línea de estudios, indagando sobre el sentido que los sectores populares atribuyen a sus decisiones y elecciones educacionales referidas a sus hijos, poniendo especial atención a las eventuales diferencias que pudieran caracterizar segmentos al interior de la clase baja. Ese es el propósito de este trabajo.

\section{Método}

Con el objeto de comprender las razones, motivos y significados asociados a la elección de escuela en sectores de clase baja, implementamos una aproximación de carácter cualitativo (Canales 2014; Taylor y Boigdan 1987) ${ }^{1}$. Para producir la información requerida, utilizamos dos técnicas complementarias: Grupo de Discusión y Entrevista Focalizada. El grupo de discusión apunta a conocer la "moral grupal"; en nuestro caso, permitió acceder a la representación social de lo que se debe o no se debe hacer al seleccionar la escuela, y los modos en que los sujetos lo interpretan

El estudio mayor del que éste forma parte indagó también en familias de clase alta y media de Santiago, y en familias de diferente nivel socioeconómico de dos agro-urbes de la zona central y sur del país. En la sección final hacemos algunas comparaciones al respecto. 
(Ibáñez 1979; Canales 2006). Las entrevistas focalizadas permitieron conocer las prácticas decisionales, y las razones y motivos del sujeto al elegir escuela (Alonso 1996; Valles 2015).

Decidimos estudiar a familias de clase baja en un sector urbano-popular prototípico de la periferia de ciudades metropolitanas, en la zona sur de Santiago, incluyendo poblaciones tradicionales (algunas surgidas de tomas de terreno), y villas y blocks más recientes. Identificamos padres que trabajan en servicios personales, economía de subsistencia o informal, y cuyos ingresos les ubican en, o levemente por sobre, las definiciones oficiales de pobreza. Al interior de estos barrios, quisimos cubrir una amplia variedad de situaciones, profundizando en aquellas más características de este sector social. El trabajo de terreno se realizó durante 2014.

Comenzamos por realizar 12 entrevistas en profundidad a madres (10) o padres (2) con hijos estudiando en diversos establecimientos, públicos y/o privados subvencionadas (varios padres tenían o habían tenido hijos en ambos tipos), tanto de enseñanza básica como secundaria. Las entrevistas indagaron sobre la propia historia educacional del padre/madre, su conocimiento y valoraciones sobre las escuelas de la zona, su experiencia durante el proceso de elección de escuelas, las razones, motivos y criterios puestos en juego para tomar las decisiones, las opciones descartadas, sus expectativas educacionales futuras para sus hijos, así como su visión y percepción sobre la heterogeneidad de las escuelas y la situación educacional general. Además, para obtener informacióndecontextosobreladinámicaescolar de la zona, entrevistamos cuatro informantes clave (funcionarios municipales y directores de escuela). Luego de esta primera fase de terreno, y siguiendo la lógica del "diseño en proceso" propio de este tipo de estudios (Canales 2014), profundizamos en los significados asociados a dos situaciones características, para lo cual realizamos los grupos de discusión: uno con padres cuyos hijos asistían a escuelas básicas públicas (la "elección" menos comprensible desde la literatura disponible y, sin embargo, más extendida en este sector social) y otro con padres cuyos hijos realizaban estudios técnicoprofesionales en la enseñanza media, dado que en nuestras indagaciones iniciales esta opción apareció simbólicamente dominante para las familias de esta clase social (lo que coincide con la distribución de la matrícula)².

El análisis del discurso producido trabajó sobre los dos ejes de la coherencia de significado (Taylor y Bogdan 1987; Ibáñez 1979). Por una parte, la identificación de paradigmas, que se pueden entender como paráfrasis o metáforas recíprocas, es decir, un conjunto de elementos que redundan sobre un eje de significados y los despliegan en sus múltiples formas; por otra, la identificación del sintagma, o la coherencia lógica y expresiva que pueda haber entre los paradigmas identificados. La coherencia resulta así en la organización de los efectos de sentido en categorías (temas) y secuencias (partes) que, como conjunto, conduzcan en una misma dirección o sentido. Cada grupo y cada entrevista fueron codificados y analizados como una unidad; posteriormente, el análisis buscó la comprensión profunda del acto de

\footnotetext{
Por cierto, hay en esta decisión razones de foco y economía del estudio; en futuras investigaciones sería recomendable profundizar también en la racionalidad que sustenta la opción de un liceo científico-humanista y una escuela privada subvencionada en estos sectores. Al presentar y discutir los hallazgos hacemos algunas menciones al respecto.
} 
elegir escuela. El análisis de conjunto permitió la elaboración de dos "tipos ideales" de elector de escuela de clase baja; los resultados presentan el material empírico, fundamentalmente para describir y comprender esta tipología. Por cierto, en la realidad (individual y grupal) los elementos identificados se presentan con matices, tensiones e intersecciones, pero nuestro propósito fue descubrir el sentido común que les anima y distingue, el cual describimos de un modo estilizado, que permite contrastarles.

\section{Resultados}

Esquemáticamente, hemos encontrado dos aproximaciones básicas a la elección de escuela entre las familias de clase baja. La primera se corresponde con las imágenes y disposiciones tradicionales referidas a la educación pública, siendo la elección de escuela menos relevante; la segunda, en cambio, participa activamente de una particular dinámica de mercado al escoger el establecimiento educativo. Estos "tipos ideales" no deben ser interpretados como electores concretos, sino como lógicas de acción que en la realidad se entremezclan. Esto se hizo claro en el análisis cuando los padres se enfrentaban a la decisión sobre la educación secundaria: la valoración de la educación técnico-profesional cruzaba a ambos tipos ideales con relativamente el mismo sentido, atravesando los significados de la elección de escuela como institución ${ }^{3}$. Por cierto, la incorporación de la dimensión curricular en la

En términos analíticos, es importante aclarar que cada padre/madre reporta visiones sobre diferentes situaciones (porque tiene hijos en diferentes colegios, reflexiona sobre el pasado de sus hijos, y expresa sus visiones sobre el resto de las situaciones que conoce), por lo que no se trata de una mera descripción de los padres como "casos" personales. decisión sobre la enseñanza media (expresada en la modalidad vocacional) no implica que las racionalidades identificadas en los dos tipos de elector desaparezcan; ellas continúan operando para decidir el establecimiento concreto a elegir. Lo interesante es ver cómo esta opción educativa tradicional, con una histórica connotación de segmentación socioeconómica en Chile, ha sido reinterpretada manteniendo su preeminencia en estos sectores, a pesar de los fuertes cambios institucionales y la enorme expansión educativa secundaria y postsecundaria. En esta sección desarrollamos estos tres elementos.

\subsection{La visión tradicional sobre la escuela: un realismo que conforma}

\subsubsection{Todas las escuelas (al alcance) son parecidas}

Los padres de sectores populares de Santiago tienen plena conciencia de ocupar un lugar subordinado en la acentuada estratificación social y de habitar territorios marcados por la pobreza, la marginalidad y la estigmatización. Los padres que poseen una visión que hemos caracterizado como "tradicional" sobre la educación no creen que esté a su alcance superar esta brecha social mediante alguna astucia ejercitada en la elección de escuela. Estos padres tienen una disposición alejada de la lógica del mercado, como un tipo de comportamiento estratégico que mejore las oportunidades educativas de sus hijos.

La "elección" de escuela de estos padres se sustenta en una visión tradicional y en cierto sentido institucional sobre la educación pública (lo que no obsta que algunos de estos padres eduquen a sus hijos en escuelas privadas 
subvencionadas gratuitas). En términos generales, creen que las escuelas (al menos aquellas a las que sus hijos pueden acceder) no presentan grandes diferencias entre sí, son todas suficientes. Ciertamente, ellos perciben que la oferta educacional ha crecido y se ha diversificado, pero se sitúan frente a este proceso con cierta distancia.

Siempre he hecho énfasis: los colegios son todos buenos, va en uno que quiera estudiar (...) en todas partes hay buenos y malos, no todo lo bueno está en un lugar ni todo lo malo en otro, está revuelto (Mujer, Municipal).

En algunos casos, esta creencia es luego confirmada a partir de la comparación directa de los contenidos curriculares entregados por escuelas públicas y privadas de los hijos de vecinos o familiares cercanos.

Bueno, porque yo le he visto los cuadernos de otros vecinos que van en subvencionados y comparo con los cuadernos de mi hija, que van en el mismo curso, es la misma materia y la [nombre de la hija] sabe lo mismo que él que es mateo en su colegio (Mujer, Municipal).

Por cierto, estos padres saben que en las escuelas de sus barrios existen alumnos con problemas sociales y conductuales, inclusive riesgosos, pero no sienten que constituyan una amenaza significativa a la formación de sus hijos. Como son parte de sus entornos, estos potenciales compañeros de sus hijos pueden desencadenar experiencias negativas -que en efecto muchos han vivido- en todos los colegios que están a su alcance, independiente de su modalidad institucional.

Yo tuve problema una vez en el [nombre de escuela], una señora grande le quería pegar a mi hijo que iba en segundo. (...) y me dice que me iba a pegar por no pegarle a mi hijo chico, me iba a pegar a mí, que yo tenía la culpa; y yo le dije '¿de qué estás hablando?'. Y la cosa es que le dije 'chao, no te conozco' y me doy la vuelta y me voy, y me pega un empujón por la espalda.
[E] ¿Era habitual eso en [nombre de escuela]?

Sí, iban personas como que no tenían mucha educación, entonces llegaban y se tiraban. Pero eso eran algunas personas, no todas, como en todas partes hay gente buena y mala.

Yo tengo un pensamiento de la educación, si yo voy a estudiar a ese colegio, no importa que sea particular, subvencionado o municipal, si me enfoco en mi educación, yo me voy a educar (Mujer, Municipal).

Más aun, estos padres son concientes de la potencial influencia negativa que -a su juiciopueden ejercer algunos compañeros de escuela sobre sus hijos y reconocen la dificultad de mantenerlos al margen de quienes perciben como pares a evitar; pero consideran una responsabilidad parental enseñar a sus hijos a conducirse en ese contexto.

Lo difícil es tratar de explicarle a tu hija que se relacione con gente que no sean hijos de delincuentes (Grupo Focal Municipal Básica).

Esta visión tradicional sobre la educación no implica ignorar las limitantes impuestas por la situación económica familiar. Las elecciones de estos padres están claramente relacionadas con sus restricciones financieras. La imposibilidad de pagar una mensualidad, transporte y en algunos casos uniformes o materiales, los conduce a optar por una escuela municipal cercana a sus domicilios. Las escuelas pagadas o que presentan algún grado de selectividad las perciben fuera de su horizonte de posibilidades.

Yo más que nada lo elegí por la cercanía, porque me queda frente a mi casa, y porque no tengo que pagar; porque los que están alrededor yo no los puedo pagar (Grupo Focal Municipal Básica).

En definitiva, los padres de este grupo no despliegan las estrategias típicas de un consumidor racional de mercado: no realizan grandes y exhaustivas búsquedas, comparan 
poco, no pagan y no manifiestan explícita disposición a cambiarse. Más que falta de interés o preocupación por la formación de los hijos, se trata de un comportamiento pragmático y realista.

Si bien, en cierto modo ellos son -o conviven con- quienes representan aquello que muchos padres intentan evitar durante el proceso de escolarización de sus hijos, y saben que, salvo excepciones, sus hijos asisten a establecimientos educacionales inferiores en la jerarquía de prestigio y calidad, en sus visiones no expresan angustia o inseguridad, sino una cuota importante de resignación.

\begin{abstract}
El colegio que iba el mayor lo cerraron (...) Por pocas matrículas y muy malo el colegio. La enseñanza no era mala, era malo el ambiente, muchos niños que venían de [indica comuna popular], otros venían de [indica población popular], unos venían de otros lados (Mujer, Subvencionado).

El [nombre de colegio] es malito, porque bueno, lo recibieron ahí (a mi hijo) siendo repitente, venía de otro colegio, y así recibe a niños de otros colegios, entonces se juntaba como lo peorcito ahí (Mujer, Subvencionado).
\end{abstract}

\subsubsection{Lo que importa es la familia y el esfuerzo individual}

Así, más que un tipo de institución (pública vs. privada, por ejemplo), para los padres que comparten esta visión, las claves para asegurar una trayectoria educativa normal, que en lo posible evite el descarrilamiento de los hijos y los ayude a una inserción básica en la estructura ocupacional, radican en el alumno y su familia: la dedicación e interés del estudiante por sus estudios, y el apoyo y formación que le puedan brindar los padres en su casa.

Para ciertas personas hay oportunidades, pero para otras no; yo creo que tiene que ver con el esfuerzo de cada uno. Mire yo no soy mucho de esas que les enseñan mucho a los niños, porque a ellos les pasan otro tipo de materias, pero yo les digo que cualquier duda está Youtube, que te enseña; trato de incentivarlos, si tienen que leer un libro, se los compro, y ahí uno ve que uno tiene que ser fundamental en la enseñanza" (Mujer, Municipal).

El influjo familiar es tan relevante, que incluso previene de las potenciales influencias negativas de los compañeros. Es decir, el contraste con la visión que se analizará en la sección siguiente -que busca la segregación precisamente para evitar este "contagio"- no está en ignorar el riesgo que asocian a algunos compañeros, sino en que estos padres confían en la potencia socializadora de la familia para prevenirlo.

Lo que pasa es que el colegio no es malo, sí hay algunos chiquillos que no son tan buenos, que hacen harto desorden, pero depende de uno cómo los enseña para que no se les peguen las malas costumbres; mi nieta nunca ha andado en cosas raras (Mujer, Municipal).

Complementariamente, los hijos son los responsables últimos de esforzarse y aprovechar las oportunidades de estudiar que, como hemos visto, se presentarán grosso modo en cualquier institución educacional. Esta responsabilización individual del logro/fracaso escolar opera a lo largo del rango de desempeño educativo. Así, creen que los estudiantes con capacidades académicas sobresalientes, aunque sean pobres e independiente de donde hayan estudiado, pueden llegar a ser profesionales universitarios, por la vía de acceder a un liceo público "emblemático". Igualmente, cuando se tiene mal desempeño, hasta la expulsión del propio hijo está justificada.

Lo echaron del [nombre de escuela], pero yo no hablo mal del [nombre de escuela] porque echaron a mi hijo, porque mi hijo era flojo. Y hasta la fecha: salió de $4^{\circ}$ medio con los Carabineros, le digo yo (Mujer, Subvencionado).

Por cierto, esta visión tiene su complemento en la generalizada expectativa de que el camino más seguro para sus hijos es acceder 
a una educación secundaria vocacional, orientada al trabajo. Así las cosas, si las escuelas funcionan de un modo básico, apenas suficiente, probablemente cumplirán su misión de canalizar a los hijos hacia el liceo técnicoprofesional y descubrir los eventuales talentos. En efecto, si para los primeros (la inmensa mayoría) se requiere una certificación básica y para los segundos (los excepcionales), bien poco porque "aprenden solos", la exigencia propiamente educacional hacia las escuelas en cierto modo se relaja.

\subsection{La intranquilidad de (tener que) participar en los márgenes del mercado}

\subsubsection{Un barrio, dos tipos de familias}

La segunda configuración prototípica respecto de la elección escolar que identificamos entre los padres de este segmento social también surge de una nítida consciencia de habitar los márgenes inferiores de la estructura social, en una zona geográfica marcada por la pobreza, y el estigma social de la violencia, la delincuencia y el tráfico de drogas; sin embargo, a diferencia del caso anterior, estos padres buscan activamente proveer a sus hijos espacios de socialización que eviten el peligro de marginalización y conduzcan hacia una integración social adecuada. El miedo frente a los peligros que implica la socialización en entornos de exclusión social y la búsqueda de ambientes escolares seguros son, por tanto, los dispositivos que movilizan a este elector de clase baja.

Estos padres consideran que, en los barrios que habitan, una parte importante de familias no tiene capacidad o bien interés de brindar a sus hijos una formación que les permita una inserción si quiera precaria en la estructura social. La clave de sus elecciones está en evitar a los hijos de estas familias. En concreto, marcan una clara diferencia entre sus hijos y los de aquellos que no trabajan, a quienes perciben fundamentalmente como delincuentes o narcotraficantes.

Hay un liceo en [comuna popular], malísimo, puras drogas, no voy a meter a mi hija ahí, porque yo, mal que mal, mis cabros son tranquilos; entonces no puedo mezclar a mis hijos con ellos, porque en mi casa no hay drogas, nadie toma, no hay ninguna de esas cosas, entonces yo no los puedo mezclar a mis hijos con ellos (Mujer, Municipal).

Evitan el contacto con todo aquel que exprese rasgos de marginalidad asociada a la violencia, drogadicción y precocidad sexual. También rehúyen de aquellos padres que viven la pobreza de manera "indigna", descuidando su presentación personal o haciendo uso de un vocabulario vulgar.

La tuve que sacar porque el ambiente era malísimo (...) Claro y las niñitas (en $8^{\circ}$ ) ya están con guagua (Mujer Subvencionado).

Son particularmente críticos con los padres que -a su juicio- no manifiestan mayores expectativas en las carreras escolares de sus hijos, y no adoptan una actitud de búsqueda de mejores oportunidades y de involucramiento directo en los asuntos escolares. Se sospecha de ellos una despreocupación por los hijos y conformismo con la posición social que ocupan actualmente.

Yo creo que acá en la comuna hay como dos tipos de apoderados, así como bien en general: están los que quieren algo mejor, y que sus hijos puedan ser algo más que ellos y ojalá salir de la comuna, y los que creen que está bien terminar cuarto medio y buscar trabajo (Mujer, municipal y subvencionado).

En definitiva, las diferencias que establecen estos padres con las familias que buscan evitar no son propiamente económicas, sino más 
bien culturales y morales; están asociadas a conductas y valores. Es una suerte de diferenciación estamental al interior de una misma clase o posición en la estructura social.

Podemos ganar lo mismo, podemos vivir en la misma cuadra, pero lamentablemente se nota de donde tú vienes, o sea, si vienes de una familia bien constituida. (...) yo creo que es una formación cultural (Hombre, Subvencionado).

\subsubsection{Elección escolar como protección social}

Dada esta percepción de riesgo social en sus entornos, estos padres intentan resguardar a sus hijos, llevándolos a estudiar fuera de sus barrios de residencia, especialmente durante la educación media, idealmente a comunas de ingresos más altos, donde la variabilidad de la oferta escolar es mayor y no carga con el estigma de la marginalidad. Así los alejan de los peligros del entorno, a la vez que favorecen su socialización con estudiantes de realidades sociales menos empobrecidas.

\footnotetext{
La gente que queremos un futuro mejor pa' los hijos tenemos que sacarlos de aquí de la zona, no podemos mandarlos a estos colegios (Mujer, Subvencionado).

En [comuna popular] hay mucha drogadicción, que no digan que no, sí hay, entonces uno tiene que sacar un poco a sus cabros de ahí, llevárselos a estudiar a otro lado (Mujer, Municipal).
}

Otra estrategia, ciertamente complementaria, es acceder a escuelas pagadas, con algún grado de selectividad. Ambos mecanismos, el copago y la selección, operan como barreras protectoras frente a la posibilidad de que estudiantes considerados riesgosos formen parte de la comunidad educativa. No es que la escuela tenga férreas políticas y complejos procesos de admisión, ni que los aranceles sean muy elevados, pero sí que pueda deshacerse de los estudiantes que son considerados una amenaza.

El hecho de que la escuela no sea gratuita impediría la entrada de alumnos de familias socialmente marginales, que no demuestran interés en pagar por la educación de sus hijos. Para estos padres, el pago por concepto de escolaridad está asociado a un valor simbólico de esfuerzo y sacrificio, y es propio de las familias que buscan mejores oportunidades, distinguiéndolas en términos morales.

Los colegios como que van así: si uno paga más, la calidad de gente es mejor, si uno paga menos, también va bajando la categoría de la gente (Mujer, Subvencionado).

Asimismo, que la escuela pueda excluir a algunos estudiantes por razones disciplinarias, o en menor medida de rendimiento, es un signo de que a ella no asisten los alumnos expulsados de otros establecimientos por motivos académicos o conductuales y que ninguna otra institución quiere recibir.

De la municipalidad mandan órdenes para que reciban a cualquier niño, lo que te decía yo, 'lo que bota la ola', y yo tenía una sobrina que estudiaba ahí y el colegio no era malo, hasta que recibieron a un montón de niños de un montón de lados y eran todos de [nombre de barrio estigmatizado como marginal] que los habían echado (Mujer, Subvencionado).

Los padres de este grupo perciben que a partir de estas barreras, las escuelas y las familias se ordenan en el mapa escolar según su nivel socio-cultural y moral, lo cual resulta en un sistema educativo altamente estratificado. Por lo pronto, es notorio que en estos contextos la escuela pública, al no contar con ningún mecanismo de exclusión, se confunde con las familias, la segregación social y el estigma; es por tanto inevitablemente riesgosa y debe en lo posible ser descartada. 
Los niños que van a colegios municipales generalmente son gente de más escasos recursos y los que van a los colegios subvencionados son gente que o puede pagar o hacen un esfuerzo, aunque sea gente más pobre, hacen un esfuerzo para que sea mejor la educación; porque todos en el fondo piensan que un colegio municipal no es bueno (Mujer, Subvencionado).

Sin embargo, que la escuela sea privada subvencionada tampoco garantiza mucho: debe ser con copago. Estas familias de clase baja están dispuestos a pagar montos que comúnmente les implican realizar grandes sacrificios económicos. Y aun así los padres sienten el deber de mantener una vigilancia activa, por cuanto los bajos aranceles no son una barrera infranqueable para el elevado riesgo ambiente en que se perciben viviendo. Hay cierta consciencia práctica de que se trata de un espacio de la sociedad con un nivel de ingreso relativamente homogéneo y cambiante, por tanto, el copago en sí mismo debe ser acompañado con otras acciones para que la protección que ofrece la segregación funcione establemente.

Son particular subvencionado, todos, (...) esos son los mejores que yo ubico (...) Bueno, en rendimiento, el ambiente, porque uno busca el ambiente, lo mejor pa' sus hijos, por eso buscamos algo mejor, o sea, algo pagado donde tuvieran mejor ambiente (Mujer, Subvencionado).

\subsubsection{La elección en la práctica: mucha oferta, pocas opciones}

En la práctica, la elección es generalmente la resultante del ajuste entre el precio de la escuela y su distancia al hogar. La cercanía del establecimiento se vuelve fundamental cuando se trata de niños pequeños, pues permite tener mayor control y seguridad, además de significar un ahorro en los costos de transporte.

Aunque algunos padres hacen también referencias a aspectos propiamente educacionales o pedagógicos, lo cierto es que no dominan su discurso. Son tenues matices en una imagen mucho más determinada por el control disciplinario y las distinciones socio-morales arriba descritas. La calidad educativa para estos padres está más bien ligada a los procesos selectivos, al tipo de familias y al ambiente, que al proceso de enseñanza-aprendizaje. En efecto, esto se llega a formular explícitamente:

En el fondo, yo pagué por el ambiente, pero no pagué por la educación (Mujer, Subvencionado).

En el proceso de búsqueda, estos padres tienden a recurrir a fuentes cercanas como amigos, familiares y vecinos, o bien, a sus propias experiencias como estudiantes y apoderados en escuelas del sector. También es frecuente que realicen evaluaciones personales y directas de las escuelas, a través de la observación de la conducta de los estudiantes y su apariencia en horarios de entrada o salida. Son padres que en general no tienen carreras escolares extendidas o exitosas, por lo que sus criterios de evaluación y conocimiento del campo son igualmente limitados.

Dadas sus restricciones económicas, encontrar una escuela que sea elegible no es sencillo, puesto que debe ser además aceptable en términos de composición social y hallarse a una distancia accesible. Paradojalmente, a pesar de observar un aumento en la oferta de escuelas, muchos padres manifestaron tener una sensación de escasez, es decir, que sus opciones reales son muy pocas y a veces únicas dentro de sus territorios.

[E]: $Y$ en caso de que algo hubiese pasado, no sé, por algún problema no hubieses podido seguir en ese colegio, ¿qué otra alternativa tenías?

Ninguna (Mujer, Subvencionado). 
En efecto, frecuentemente las limitantes de la oferta y del presupuesto familiar les impiden matricular a sus hijos en las escuelas deseadas, debiendo hacerlo en otras que ellos mismos desestiman. En cuanto la economía familiar mejora, estos padres no dudan en cambiar a sus hijos a una escuela particular subvencionada con copago. Así, las fluctuaciones en la disponibilidad de dinero explican la alta movilidad escolar de sus hijos.

\begin{abstract}
Yo puse a mi hija en el Colegio [Part. Subvencionado 1], pero por temas económicos tuve que sacarla y la cambié al [Municipal] que es de la comuna, pero es pésimo, es un colegio malo, para mí fue malo. Después volví y la coloqué en el colegio [Part. Subvencionado 2], particular subvencionado, porque el otro era particular. (...) La cambié por problemas económicos, porque no tenía pa' pagar y después se me mejoró la situación y la traje a [Part. Subvencionado 2] (Mujer, Municipal "Emblemático").
\end{abstract}

En consecuencia, cuando estos padres no tienen la posibilidad de acceder a una escuela privada subvencionada pagada, viven la experiencia educativa de sus hijos con gran frustración e intranquilidad. Como si estuviesen incumpliendo su deber básico de darles una educación adecuada que conduzca a alguna forma de integración social, y evite el extravío moral y la marginalización. Frente a la percepción de habitar un "mercado escolar" sumamente restringido, donde las opciones de escuelas válidas y accesibles son escasas, estos padres reclaman un particular subvencionado como carencia y necesidad; como una demanda, casi un derecho legítimo.

Yo creo que todos nos sentimos de alguna manera impotentes de no tener a nuestros hijos en un colegio mejor, yo creo que a todos nos gustaría tener a nuestros hijos en un colegio particular, que te enseñen bien, porque los colegios municipales, no digo que todos, pero el niño que cachó, cachó y el que no, se queda atrás no más (Grupo Focal Municipal Básica).
M1: Deberían poner más colegios subvencionados, pero para la gente pobre...más accesibles (Grupo Focal Municipal Básica).

\subsection{La salida sensata: elegir educación media Técnico Profesional}

Es interesante notar que los padres de sectores populares, aunque como hemos visto difieren en su apreciación sobre la educación primaria, tienden a coincidir en su visión curricular sobre la educación secundaria: para la mayoría de sus hijos, prefieren la educación vocacional por sobre una preparación general. Así, la enseñanza media la escogen en base a expectativas educacionales realistas, de corto alcance, históricamente asociadas a los sectores populares: que los hijos adquieran una educación técnica secundaria, para aumentar sus posibilidades de empleabilidad y asegurar una inserción rápida en el mercado laboral.

Los más pobres, para quienes la continuidad de estudios aún se ve lejana e incierta al no disponer de recursos económicos suficientes o estables para garantizar el pago de una educación postsecundaria, evalúan como una gran ventaja que la enseñanza media califique directamente para el trabajo.

\footnotetext{
Yo soy partidaria de que el niño vaya a un industrial o a un comercial, porque el niño sale de $4^{\circ}$ medio, sale sabiendo algo, en cambio de un liceo, no sale sabiendo nada. Yo voy por lo siguiente, por lo práctico, yo no le puedo pagar una universidad, por más esfuerzo que haga, yo jamás voy a poder pagar una carrera en una universidad, entonces yo digo, se va a perder (Mujer, Municipal).
}

En términos más amplios, la elección de la educación técnica secundaria se justifica cuando los hijos no han tenido un desempeño académico destacado que les permita postular a un liceo público académicamente selectivo 
y transitar con ventajas hacia la educación superior.

A mí me hubiera gustado que mi hijo fuera a un colegio emblemático, pero él no tiene tan buenas notas... me hubiera encantado que hubiese ido al [liceo público tradicional académicamente selectivo] (Grupo Focal Técnico-Profesional).

En enseñanza media, la deseabilidad de que los hijos estudien en liceos públicos "emblemáticos" es casi universal entre estos padres. Es esta la vía más segura para arribar a la universidad. Sin embargo, es un camino solo viable para los estudiantes de destacado desempeño y talento.

\footnotetext{
Muchos se quedan en la villa, porque pa' entrar a un colegio emblemático, te piden nota, te piden dar una prueba, que pasen la prueba (...) Claro, que tengan mejor rendimiento (Mujer, Subvencionado).
}

La impotencia inicial de estos padres por no poder acceder a una promoción más segura a la educación terciaria se compensa con una clara racionalidad. Los padres que matricularon a sus hijos en la educación técnica afirman el sentido de esta elección: que la educación técnica conduce a algún lugar (a diferencia de la científica humanista en los liceos que son accesibles para ellos) y permite, por tanto, pasar de la frustración a la conformidad con sus decisiones.

Es que yo pienso que todos los papás queremos que los hijos sean profesionales, pero mirándolo ahora, fríamente, yo pienso que lo más fácil es un técnico, y por la parte económica (Grupo Focal Técnico-Profesional).

En efecto, quienes eligen la educación técnica profesional valoran la noción de trabajo en su expresión más clásica. El sentido es entregar a los hijos algún tipo de conocimiento y herramientas para que, en un futuro próximo, se inserten de mejor forma en la estructura laboral. Se busca evitar la condena mayor que es el trabajo no-calificado, al cual se somete quien queda sin título ni especialidad.

Yo le he explicado, que con esos conocimientos en Contabilidad, incluso puede trabajar por cuenta de él. (...) yo encuentro que es importante que a los niños les den una profesión, que salgan encaminados en algo (Grupo Focal Técnico Profesional).

La novedad de esta misma decisión respecto de las décadas anteriores es que ya no condenaría a sus hijos al trabajo irreversiblemente simple. Ahora el técnico puede ser visto como el primer paso, el primer título que les permite participar en la estructura social, y a partir de ahí, con la seguridad que da una certificación, visualizar otros futuros y expectativas, ahora ligadas al estudio de una carrera superior.

\footnotetext{
Que él saque un técnico, tenga un título y si él quiere seguir en la universidad, lo vamos a apoyar (Grupo Focal Técnico Profesional).
}

La enseñanza media técnica profesional no niega entonces las posibilidades de continuar estudios a futuro, más bien la reafirma, pues es vista como un mecanismo que permite adquirir las herramientas para insertarse en el mundo laboral y así poder conseguir los recursos para financiar una carrera superior.

Yo creo que es para tener una herramienta de trabajo digamos lo más luego posible, empezar a trabajar primero y después a lo mejor se les da seguir estudiando (...) e ir subiendo su nivel. Yo creo que hay harta gente que lo hace así, más de un 60\% (Mujer, Subvencionado).

La sensatez de esta opción se ve reforzada por un cierto discurso público, que perciben estos padres, de revalorización de la educación técnica, basado en el hecho de que en Chile "sobran profesionales y faltan técnicos", producto de la enorme masificación reciente de las universidades. Así, la opción de estudios técnicos pareciera tener el futuro de su lado, por 
lo que quizás supere el estigma social que ha llevado.

Pero si usted ve, en los diarios, en todos lados, se necesitan más técnicos que profesionales, que ingenieros y todo eso (...) De repente se postulan ingenieros en ejecución en algo y técnicos y necesitan un técnico, no un ingeniero, porque al ingeniero le van a tener que pagar más (Grupo Focal Técnico Profesional).

En suma, la educación técnica puede entenderse como la salida pragmática que encuentra este estrato, frente a lo que perciben como una condena de la educación media científica humanista a su alcance, que no conduce a la Universidad ni califica para el trabajo. Más aun, elegir educación técnica ya no implica resignar anticipadamente al sueño promocional: para estos padres, la modalidad técnico profesional se levanta hoy como una primera conquista, el título inicial que hace viable una carrera mayor por el acceso a la anhelada formación terciaria, ya sea en institutos profesionales, centros de formación técnica o incluso en carreras universitarias menos selectivas.

\section{Discusión y conclusiones}

Del análisis identificamos básicamente dos tipos de electores: uno activo e intranquilo, otro tradicional pragmático; se diría que los primeros están luchando en la parte inferior del mercado, en sus márgenes; mientras los segundos permanecen fuera de él. Adicionalmente encontramos una fuerte imagen social de la elección-paraalgunos laúnicareal-deeducación técnica-secundaria. Cada una de estas "elecciones" tiene para los padres de sectores populares una densa trama de significados, muy lejos de la caracterización pasiva del "no elector" tan extendida en la literatura. En esta sección final proponemos algunos elementos conceptuales para interpretar esta evidencia y ponerla en perspectiva.

De acuerdo a nuestros hallazgos, el núcleo de la visión del elector tradicional de sectores populares está en que considera que no existen diferencias relevantes entre las escuelas a las que sus hijos pueden acceder; es la subsistencia (aunque algo erosionada) de la confianza básica hacia la institucionalidad educativa. De hecho, es destacable que entre estos padres encontramos algunos que manifiestan confianza y valoración hacia la educación pública que reciben del estado. Por sus limitaciones económicas, no consideran "elegibles" las escuelas que cobran o no se muestran convencidos de que ese pago haga una diferencia; reconocen que algunos alumnos son indisciplinados y hasta riesgosos, pero creen que esas malas experiencias ocurren en todos los ambientes; se han informado que no todas las escuelas son accesibles, por ello se conforman cuando "encuentran un cupo" para sus hijos; tienen plena conciencia de que ocupan un lugar subordinado en la marcada estratificación social chilena, pero no creen que esté a su alcance vencer esa brecha socioeducativa. Más que resignación, pragmatismo. De hecho, lo que estos padres tratan de optimizar es el principio del esfuerzo individual: buscan agenciar el futuro de sus hijos de manera familiar, con las herramientas y recursos que cada uno logra gestionar. Sin hacer muchas comparaciones, con menos movilidad y sin pagar por la educación de sus hijos, estos padres están "fuera del mercado" educacional. En efecto, ellos están donde muchos otros no quieren y son (o conviven con) quienes los demás quieren evitar durante la formación de sus hijos.

La visión de estos padres de sectores populares de Santiago es similar a la que prevalece 
en padres de bajos ingresos habitantes de agro-urbes que han tenido procesos de modernización menos intensos. Dichas familias expresan muy claramente la idea clásica de la educación pública como camino legítimo, y luego encarnan esta visión en su relación con el sistema educacional. En este camino depende de uno mismo y de la familia tener un rendimiento suficiente, no se aspira a mucho más. No hay por tanto mayor "elección escolar" salvo el camino técnico (agrícola, deseablemente), que está en sí mismo naturalizado como el único posible. Incluso, llama la atención en estos sectores cierta crítica o desconfianza hacia los establecimientos particular-subvencionados, sobre todo los de reciente creación: no habrían mayores razones para pagar por un servicio que, otorgado por el Estado, es gratis y de calidad aceptable (Bellei, Canales y Orellana 2015).

Estalógicadelasfamiliasquehemosdenominado "tradicional" expresa la pervivencia en los sectores populares de los elementos básicos de la visión institucional de la mayoría de los sistemas educacionales modernos, incluyendo el chileno previo a la reforma de mercado (Bellei 2015). En lo sustantivo, las escuelas no debieran diferenciarse, por cuanto se espera que las oportunidades de aprendizaje sean similares en cualquier institución; "elegir" entre escuelas (por lo demás públicas y gratuitas), carece así de sentido. El estado, no el mercado, es el garante en este arreglo. Esta "ideología" de la educación de masas se completaba con una concepción fuertemente estratificada del logro educacional, basado principalmente en el mérito (talento y esfuerzo) individual. Para los padres de clase baja que estudiamos, la presencia de liceos públicos académicamente selectivos encarna la viabilidad de esta angosta puerta a la movilidad social amplia y produce por tanto un efecto simbólico de coherencia a esta visión. La estratificación social de la carrera educacional completó su institucionalización en Chile por la vía de legitimar y expandir la educación secundaria técnico-profesional para los sectores pobres, proceso que se encuentra plenamente vigente. Así las cosas, estas familias han permanecido relativamente al margen del "mercado educacional" desarrollado en Chile. En efecto, la oferta de escuelas privadas subvencionadas, especialmente aquellas con copago, no se expandió en estos sectores sino hasta muy recientemente. Más aun, en lo esencial, estas familias confían en que su poder socializador mantendrá a sus hijos a salvo de los riesgos de vivir en zonas urbano-populares, desechando así lo que perciben como la principal promesa del sector privado.

Nuestro estudio también identificó el desarrollo de una visión alternativa a esta tradicional, portada por padres de sectores populares que están participando activamente del dinámico mercado escolar chileno, con la consciencia de ubicarse en una posición muy subordinada en el estratificado sistema educacional. Estas familias creen, por tanto, que la elección de escuela es muy importante para la adecuada integración social de sus hijos. La clave de sus decisiones es buscar las instituciones que permitan evitar a los hijos de familias que creen riesgosas; la actitud dominante no es de discriminación, socio-fobia ni menos "arribismo", sino de reconocimiento responsable de los riesgos que implica la socialización en entornos de exclusión social. Así, prefieren escuelas capaces de excluir (porque cobran o seleccionan) y evitan escuelas "basurero" donde se concentran quienes han tenido mala conducta o crónico mal desempeño. Aunque la distinción municipal/particular subvencionada es relevante, para la mayoría 
de estos padres es insuficiente: la escuela municipal es casi seguramente riesgosa, pero hay excepciones; y la privada subvencionada no garantiza nada por sí misma, porque sus (bajos) aranceles no son una sólida barrera para el elevado riesgo ambiente. En consecuencia, con una fuerte orientación sociocultural en sus decisiones, estos padres tienen una conducta activa de búsqueda, elección y vigilancia sobre las escuelas. Incluso, algunos padres buscan opciones fuera de su zona de residencia, para que sus hijos interactúen con compañeros de otras realidades sociales. Por cierto, dadas sus limitaciones económicas muchas veces experimentan la frustración de matricular a sus hijos en establecimientos que descalifican, y muchos de sus cambios de escuela en sentido "ascendente" y "descendente" están asociados a fluctuaciones presupuestarias en la frágil economía familiar, por lo que viven en una permanente sensación de riesgo.

Es precisamente una percepción exacerbada del peligro social circundante lo que creemos anima la intranquilidad de las familias de clase baja que adoptan esta aproximación activa respecto de la elección escolar, inquietud que creemos asociada con lo que ha sido conceptualizado en estudios recientes de estratificación social como "inconsistencia posicional", un sentimiento generalizado en Chile, alimentado en los sectores pobres por la fragilidad socioeconómica y las amenazas y peligros urbanos (Araujo y Martuccelli 2011). Pagar (aunque sea poco) y preferir escuelas con cierta capacidad de exclusión (i.e. evitar las escuelas públicas) es visto como una forma de clausura social que permite distanciarse de familias percibidas como marginales, protegiendo a los hijos. Se trata de la versión popular de un esquema de percepción y acción identificado como dominante al interior de los sectores medios emergentes, base de la dinámica de oferta-demanda de las escuelas privadas subvencionadas con copago (Canales, Bellei y Orellana 2016). La búsqueda de la "decencia" para sobrellevar la situación de pobreza, en contraposición a una actitud indigna que arriesga degradarse socialmente hacia el alcoholismo, la delincuencia o la drogadicción, resulta enormemente significativa para estos padres. La relación conflictiva de este sustrato cultural con las políticas sociales focalizadas ya había sido advertida por Martínez y Palacios (1996). Según estos autores, al interior de los sectores populares esta actitud marca una diferenciación estamental: en un contexto de política social focalizada, el pobre "decente" busca distanciarse de los bienes y servicios distribuidos gratuitamente por el estado, percibidos como "caridad", cuyo estigma pone en duda el esfuerzo familiar; el pobre decente no espera regalos, trabaja para comprar. Nuestros hallazgos sugieren la extensión de esta lógica a la educación. Nótese que en un segmento social en que las diferenciales de ingreso son menores y en que la precaria economía familiar les hace transitar "alrededor" de la pobreza constantemente (Castro y Kast 2004; Ruiz Encina y Boccardo 2014), estas distinciones simbólicas asociadas a pequeñas variaciones de precio-selectividad de las escuelas pueden adquirir enorme valor para las personas.

La noción de peligro que gatilla la elección de estos padres resulta mucho más intensa que la que encontramos en familias igualmente de escasos recursos, pero en ciudades no metropolitanas, un hallazgo igualmente consistente con el mencionado estudio de Araujo y Martuccelli (2011). Para algunas familias de ciudades de provincia, la experiencia o la percepción de 
los fenómenos urbanos emergentes -como la delincuencia o la drogadicción- ponen en riesgo la disciplina y la cohesión propia de la vida tradicional popular, haciendo deseable optar por colegios particular-subvencionados de rango inferior de precio, o incluso gratuitos, donde los segmentos peligrosos no logran llegar o si llegan no amenazan la sociabilidad buscada. Sin embargo, aunque igualmente orientada por un argumento social antes que académico, esta experiencia no se da con el trauma ni el dolor de lo visto en la capital, pues no amenaza el proyecto de vida, ni pone en duda la integración social que experimentan (Bellei, Canales y Orellana 2015).

Como se aprecia, la racionalidad que subyace a ambas lógicas de elección escolar se ancla en horizontes normativos altamente constituyentes de los sujetos, y que guardan una importante continuidad histórica en el segmento bajo de la sociedad chilena. En este sentido, es muy interesante que los padres de sectores populares tiendan a converger en su visión sobre la mejor opción para la educación secundaria (aunque no necesariamente en el establecimiento concreto a elegir). Contra el telón de fondo de los liceos públicos "emblemáticos", quimera solo reservada para sus (improbables) hijos académicamente talentosos destinados a ser profesionales, se impone la opción prudente de la enseñanza vocacional. Por cierto, se trata de una naturalización de la histórica segmentación socio-educativa, con fuerte base en la demanda social y la institucionalidad educacional (Errázuriz y Martinic 1994; Bellei 1996). Más aun, en el contexto de creciente masificación de la educación post-secundaria (que incluye carreras cortas y universidades poco selectivas), la elección de liceos técnico-profesionales aparece hoy menos costosa que ayer, cuando la garantía de una rápida inserción laboral como obrero calificado se hacía a costa de sacrificar definitivamente el sueño promocional. Así, la elección de modalidad de estudios secundarios que realizan las familias de clase baja se basa en una racionalidad de especialización curricular y conexión educación-trabajo cuyas bases (ahora sí, educacionales) anteceden la reforma de mercado aplicada a la educación, todo lo cual refuerza nuestra tesis de una penetración sólo parcial de ésta en dichos sectores sociales.

En definitiva, comprender la elección escolar de las familias obliga a considerar el contexto social y de política pública, pues se trata de complejas racionalidades prácticas, que ponen en juego un denso sustrato cultural, canalizado por una institucionalidad educacional particular. Así, la modernización neoliberal chilena aplicada al campo de la educación, ha ido transformando la educación pública gratuita en una especie de "servicio de atención focalizada" administrado por los municipios, que amenaza con devenir en gueto de exclusión social. La solución de mercado (básicamente financiada por el estado) ofrece una salida privada, que a su vez segmenta por precio y diferentes grados de selectividad de alumnos-familias, reforzando la fragmentación social al interior de las comunidades locales; se ha argumentado que estas dinámicas explican en buena parte la elevada segregación socioeconómica de la educación chilena (Valenzuela, Bellei y De los Ríos 2014). Más aun, la idea de una integración social "estamentalizada" ha sido recurrente en el debate sociológico sobre el Chile actual y el surgimiento de nuevos movimientos sociales (Ruiz Encina 2015; Bellei, Cabalin y Orellana 2014). Con todo, esta visión no llega a ser dominante en los sectores populares estudiados, en donde la noción tradicional 
sobre la educación pública sobrevive como una especie de anacronismo cultural, cada vez más fuera del juego, especialmente al contrastársela con la racionalidad del consumidor de educación que la institucionalidad de mercado propicia. Por cierto, la reforma educacional en curso anuncia un revival en este sentido, expandiendo la gratuidad y controlando la selección de estudiantes. Lo que nuestro estudio sugiere es que dicha agenda de reformas encuentra más coherencia en su realización creando una institucionalidad que encarne y promueva el principio universal del derecho igualitario a la educación, es decir, la educación pública.

\section{Bibliografía}

Alegre, M. y Benito, R. 2012a. ¿En qué se fijan las familias a la hora de escoger la escuela de sus hijos? Factores de elección y descarte escolar en la ciudad de Barcelona. Profesorado, 16(3), 59-79.

Alegre, M. y Benito, R. 2012b. 'The best school for my child?' Positions, dispositions and inequalities in school choice in the city of Barcelona. British Journal of Sociology of Education, 33(6), 849-871.

Alegre, M., Benito, R. y González, I. 2014. School educational project as a criterion of school choice: discourses and practices in the city of Barcelona. Journal of Education Policy, 29(3), 397-420.

Alonso, L. 1996. Sujeto y Discurso. El lugar de la entrevista abierta en las prácticas de la sociología cualitativa. En Gutiérrez, J. y Delgado, J. Metodologías cualitativas de investigación social. Madrid: Síntesis.

Araujo, K. y Martuccelli, D. 2011. La inconsistencia posicional: un nuevo concepto sobre la estratificación social. Revista de la CEPAL, (103), 165-178.

Ball, S. 1993. Education markets, choice and social class : the market as a class strategy in the UK and the USA. British Educational Research Journal, 14(1), 3-19.

Ball, S. 2003. Class Strategies and the Education Market. Londres: Routledge.

Ball, S., Bowe, R., y Gewirtz, S. 1996. School choice, social class and distinction: the realization of social advantage in education. Journal of Education Policy, 11(1), 89-112.

Ball, S. J., Bowe, R., y Gewirtz, S. 1995. Markets, choice and equity in education. Buckingham: Open University Press.

Ball, S. y Reay, D. 1998. 'Making their Minds Up': family dynamics of school choice. British Educational Research Journal, 24(4), 431-448.

Ball, S. y Vincent, C. 1998. "I heard it on the grapevine":"hot" knowledge choice and school choice. British Journal of Sociology of Education, 19(3), 377-400.

Ballion, R. 1991. La bonne école. Évaluation et choix du collège et du lycée. Hatier, coll. Société. Paris.

Ballion, R. 1986. Le choix du college: le comportement èclairé des familles. Revue Francaise de Sociologie, 27(4), 719-734.

Bellei, C. 2015. El gran experimento. Mercado y privatización de la educación chilena. Santiago: LOM Ediciones.

Bellei, C. 1996. Equidad social y expansión de la Educación Media Técnico-Profesional.Proposiciones, 27, SUR-Profesionales, Santiago.

Bellei, C., Cabalin, C. y Orellana, V. 2014. The 2011 Chilean student movement against neoliberal educational policies. Studies in Higher Education, 39(3), 426-440.

Bellei, C., Canales, M. y Orellana, V. 2015. Informe Final Proyecto Fondecyt "El soporte cultural para el mercado escolar". Centro de Investigación Avanzada en Educación y Departamento de Sociología, Universidad de Chile.

Ben Ayed, C. 2001. Savoir, vouloir, pouvoir. Choix scolaires et désillusions des familles populaires. Revue du Centre de Recherché en Education, 20, 89-114.

Bernal, J. 2005. Parental choice, social class and market forces: the consequences of privatization of public services in education. Journal of Education Policy, 20(6), 779-792.

Bosetti, L. 2004. Determinants of school choice: understanding how parents choose elementary schools in Alberta. Journal of Education Policy, 19(4), 387-405.

Broccolichi, S. y Van Zanten, A. 2000. School competition and pupil flight in the urban periphery. Journal of Education Policy, 15(1), 51-60.

Butler, T. y Van Zanten, A. 2007. School choice: a European perspective. Journal of Education Policy, 22(1), 1-5.

Canales, M. 2014. Investigación social. Lenguajes del diseño. Santiago: LOM Ediciones.

Canales, M. 2006. El grupo de discusión y el grupo Focal. En Canales M. (ed.), Metodologías de Investigación Social. Introducción a los oficios. Santiago: LOM Ediciones.

Canales, M., Bellei, C. y Orellana, V. 2016. ¿Por qué elegir en escuela particular subvencionada? Sectores medios emergentes y elección de escuela en un sistema de mercado. Estudios Pedagógicos (en prensa).

Carrasco, A., Falabella, A. y Mendoza, M. 2015. School choice 
in Chile as a sociocultural practice. An ethnographic enquiry. En P. Seppanen et al. (Eds.). Contrasting dynamics in education politics of extremes, pp. 245-266. Sense Publishers.

Cartier, M., Coutant, I., Masclet, O y Siblot, J. 2008. La France des petits-moyens. Enquête sur la banlieue pavillonaire. París: La Découverte.

Castro, R. y Kast, F. 2004. Movilidad de la pobreza en Chile. Análisis de la encuesta panel 1996/2001 (No. 85). Santiago.

Córdoba, C. 2014. La elección de escuela en sectores pobres: Resultados de un estudio cualitativo. Psicoperspectivas, 13(1), 56-67.

Chubb, J. y Moe, T. 1990. Politics, markets, and America's schools. Brookings Institution Press.

Da Costa, M. y Koslinski, M. 2012. Escolha, estratégia e competição por escolas públicas. Pro-Posições, 23(2), 195-213.

Devine, F. 2004. Class practices: How parents help their children get good Jobs, Cambridge: Cambridge University Press.

Echols, F. y Willms, J. 1995. Reasons for school choice in Scotland. Journal of Education Policy, 10(2), 143-156.

Errázuriz, M. y Martinic, S. 1994. Demandas sociales a la Educación Media. Colección de estudios sobre Educación Media, Mineduc, Programa MECE, Santiago.

Felouzis, G., Maroy, C. y Van Zanten, A. 2013. Les Marchés scolaires: Sociologie d'une politique publique d'éducation. París: Puf.

Felouzis, G. y Perroton, J. 2009. Grandir entre pairs à l'école. Ségrégation ethnique et reproduction sociale dans le système éducatif français. Actes de la Recherche en Sciences Sociales, 5(180), 92-100

Fernández, M. 2004. Elección de escuela: Efectos sociales y dilemas en el sistema educativo público en Andalucía. Revista de Educación, 334, 377-390.

Gentili, P., Frigotto, G., Leher, R. y Stubrin, F. 2009. Políticas de privatización, espacio público y educación en América Latina. Rosario: CLACSO - Homo Sapiens.

Gewirtz, S., Ball, S. y Bowe, R. 1995. Circuits of schooling: a sociological exploration of parental choice of school in social class context. The Sociological Review, 43(1), 52-78.

Ibáñez, J. 1979. Más allá de la sociología. El grupo de discusión, teoría y práctica. Madrid: Siglo XXI.

Martínez, J., y Palacios, M. 1996. Informe sobre la decencia. La diferenciación estamental de la pobreza y los subsidios públicos.
Santiago: Ediciones SUR.

Martinic, S. y Pardo, M. (Eds.). 2000. Economía política de las reformas educativas en América Latina. CIDE- PREAL.

Navarro, L. 2004. La escuela y las condiciones sociales para aprender $y$ enseñar. UNESCO \& IIPE, Eds. Buenos Aires.

Olmedo, A. 2007. Las estrategias de elección de centro educativo en las familias de clase media. Estudio de la incidencia social en un mercado educativo local. Granada: Editorial Universidad de Granada.

Palheta, U. 2012. La domination scolaire. Sociologie de l'enseignement professionnel et de son public. París: Puf.

Raczynski, D. y Hernández, M. 2011. Elección de colegio, imágenes, valoraciones y conductas de las familias y segregación social escolar. Asesorías para el Desarrollo. Santiago.

Rambla, X. 2003. Las desigualdades de clase en la elección de escuela. Revista de Educación, 330, 83-98.

Reay, D., Ball, S. y Taylor, P. 1997. 'Spoilt for Choice': the working classes and educational markets. Oxford Review of Education, 23, 89-101.

Roland, N. 2012. Choisir une école dans les milieu populaires. Education et formation, 297, 75-96.

Ruiz Encina, C. 2015. De nuevo la sociedad. Santiago: LOM Ediciones-Fundación Nodo XXI.

Ruiz Encina, C. y Boccardo, G. 2014. Los chilenos bajo el neoliberalismo. Clases y conflictos sociales. Santiago: El Desconcierto - Fundación Nodo XXI.

Taylor, S. J. y Bogdan, R. 1996. Introducción a los métodos cualitativos de investigación. La búsqueda de significados. Buenos Aires: Paidós.

Valenzuela, J. P., Bellei, C. y De Los Ríos, D. 2014. Socioeconomic school segregation in a market-oriented educational system. The case of Chile. Journal of Education Policy, 29(2), 217-241.

Valles, M. 2015. La entrevista cualitativa. Madrid: CIS, Centro de Investigaciones Sociológicas.

Vincent, C., Braun, A. y Ball, S. 2010. Local links, local knowledge: Choosing care settings and schools. British Educational Research Journal, 36, 279-298.

Waslander, S. y Thrupp, M. 1995. Choice, competition and segregation: an empirical analysis of a New Zealand secondary school market 1990-1993. Journal of Education Policy. 10(1), 1-26. 